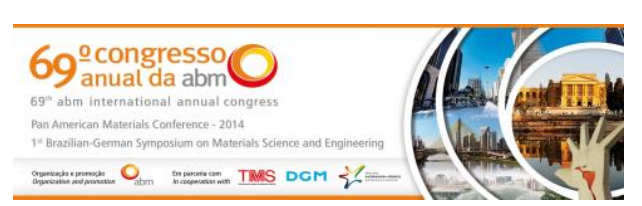

Tema: Materiais cerâmicos, compósitos e poliméricos

\title{
ANÁLISE TEÓRICO-EXPERIMENTAL DO PROCESSO DE EXPANSÃO DE EXTREMIDADES DE TUBOS DE PAREDE FINA EM MATRIZ*
}

\section{Resumo}

Alberto Moreira Guerreiro Brito ${ }^{1}$ Lirio Schaeffer ${ }^{2}$

Componentes tubulares de parede fina têm aplicações em diversas áreas industriais, na forma de trocadores de calor, amortecedores de impacto, sistemas de fixação, absorvedores de energia, pré-formas para outros processos de conformação mecânica, etc. Neste trabalho é apresentado um estudo de natureza teóricoexperimental envolvendo o processo de expansão de extremidades de tubos. Buscase determinar os parâmetros que governam o mesmo, suas limitações e se a conformação em matriz pode ser uma alternativa viável a processos mais complexos, como por exemplo, hidroconformação. É feita a caracterização do material (uma liga de alumínio ABNT 6061) através do levantamento de curvas de escoamento, perfis de microdureza Vickers, e deformabilidade quanto ao surgimento de trincas e de instabilidade plástica (flamblagem). Ao final são conduzidas simulações através de elementos finitos e experimentos do processo de expansão. São variadas características geométricas dos tubos e das ferramentas e o estado do material (diferentes tratamentos térmicos).

Palavras-chave: Conformação de tubos de parede fina; Conformação de tubos de alumínio; Expansão de extremidades de tubos; Instabilidade plástica de tubos; Dano crítico.

\section{THEORETICAL AND EXPERIMENTAL ANALYSIS OF THE ENDS EXPANSION OF THIN-WALLED TUBES USING A DIE}

\begin{abstract}
Thin-walled tubular components have applications in several industrial areas, as heat exchangers, shock absorbers, fastening systems, energy absorbers, preforms to other metalworking processes, etc. This work presents a theoretical and experimental study involving the tube end expansion process using a die. The aim is search the parameters that govern the process, its limitations and if it can be a feasible alternative to more complex processes such as hydroforming. The material (an aluminum alloy ABNT 6061) characterization is done thru the obtaining of flow curves, microhardness profiles and deformability limits related to the onset of cracks and plastic instability. The research on the expansion process is carried out thru numerical simulations and experimental tests to different tubes and tools geometries and material states (different heat treatments).

Keywords: Metal forming of thin-walled tubes; Metal forming of aluminum alloy tubes; Tube end expansion; Plastic instability of tubes; Critical damage.

1 Engenheiro metalúrgico, Doutor em Engenharia, Pesquisador, Laboratório de Transformação Mecânica, Universidade Federal do Rio Grande do Sul, Porto Alegre, RS, Brasil.

2 Engenheiro mecânico, Doutor em Engenharia, Professor Titular, Laboratório de Transformação Mecânica, Universidade Federal do Rio Grande do Sul, Porto Alegre, RS, Brasil.
\end{abstract}

\footnotetext{
* Contribuição técnica ao $69^{\circ}$ Congresso Anual da ABM - Internacional e ao 14ํㅡㄹ ENEMET - Encontro Nacional de Estudantes de Engenharia Metalúrgica, de Materiais e de Minas, 21 a 25 de julho de 2014, São Paulo, SP, Brasil.
} 


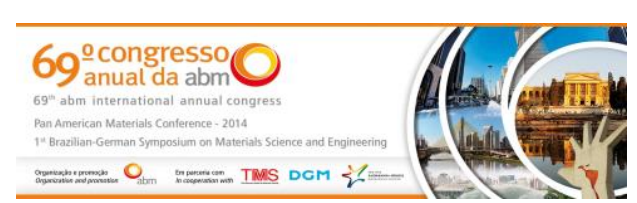

\section{INTRODUÇÃO}

Componentes tubulares de parede fina têm aplicações em diversas áreas industriais, na forma de trocadores de calor, amortecedores de impacto, sistemas de fixação, absorvedores de energia, pré-formas para outros processos de conformação mecânica, etc. Muitas vezes a produção de tais componentes envolve a execução de operações de acabamento ou fixação na extremidade dos tubos [1-3]. O objetivo deste trabalho é a realização de um estudo de natureza teórico-experimental envolvendo o processo de expansão conforme visto na Figura 1. No processo, o primeiro contato entre o tubo e a ferramenta ocorre no ponto $A$ e até o ponto $B$ existe apenas um deslizamento entre as superfícies. No ponto B começa a ocorrer o dobramento até o ponto $\mathrm{C}$. Entre os pontos $\mathrm{C}$ e $\mathrm{D}$ ocorre estiramento ao longo da direção circunferencial, $\theta$. Entre os pontos D e E ocorre um "endireitamento", sendo que no ponto $D$ o tubo deixa de ter contato com o punção. Entre os pontos $E$ e $F$ existe uma região de deformação livre onde o tubo se comporta como um corpo rígido. A influência do atrito ocorre predominantemente entre os pontos $B$ e $D$ embora alguma resistência ao movimento possa ocorrer na região cilíndrica $A B$.
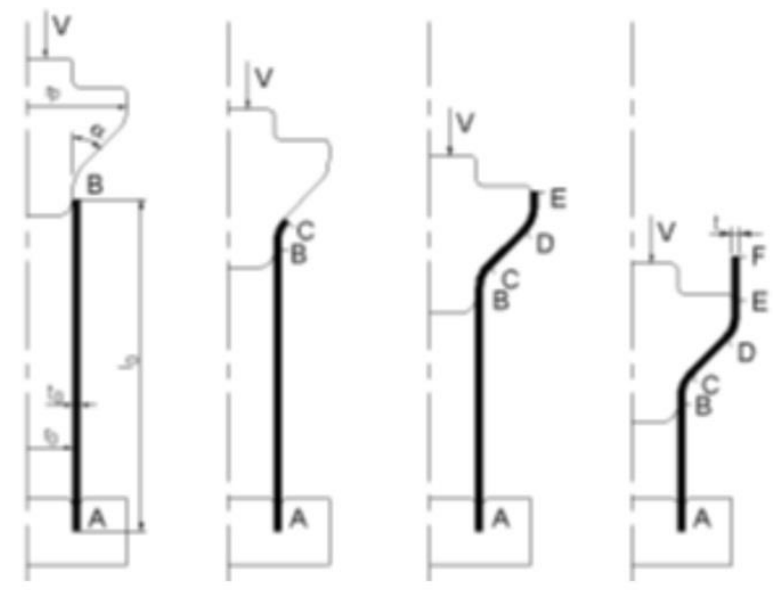

Figura 1. Representação esquemática do processo de expansão.

O processo é testado com a utilização de uma liga de alumínio ABNT 6061 recozida (estado "0"), solubilizada e envelhecida naturalmente (estado "T4") e solubilizada e envelhecida artificialmente (estado "T6"). O material, nos três estados, é previamente caracterizado através de análise química, levantamento de curvas de escoamento, microdureza Vickers e instabilidade plástica (ruptura e flambagem). Também é feita a caracterização do processo quanto às condições tribológicas.

Literatura sobre processos de acabamento de extremidades de tubos é relativamente rara. $O$ primeiro trabalho que o autor pode localizar a respeito do processo de expansão foi publicado por Avitzur [4] em 1965. Nesse trabalho é apresentado um modelo para o processo baseado na teoria do limite superior, prevendo a força necessária em regime permanente, a máxima deformação em área possível e o ângulo ótimo para a matriz. Em 1996, Sadok et al. [5] utilizam o programa de elementos finitos FORGE2 para calcular a força e distribuição da deformação efetiva ao longo da zona de deformação. No caso da força foram encontrados desvios de até $13 \%$ em relação a valores experimentais obtidos pelos autores. Almeida et al. [6] apresentam, em 2006, um estudo abrangente sobre o processo mostrando a influência do atrito sobre o fluxo de material e sobre os efeitos

\footnotetext{
* Contribuição técnica ao $69^{\circ}$ Congresso Anual da ABM - Internacional e ao 14ํㅡㄹ ENEMET - Encontro Nacional de Estudantes de Engenharia Metalúrgica, de Materiais e de Minas, 21 a 25 de julho de 2014, São Paulo, SP, Brasil.
} 
do dano e dos caminhos de deformação sobre a ocorrência de trincas, enrugamento e flambagem.

\section{MATERIAIS E MÉTODOS}

Os corpos de prova para levantamento de curvas de escoamento, calibração do atrito e calibração do dano foram obtidos a partir de uma barra extrudada, enquanto que as amostras para teste do processo em si foram cortadas a partir de um tubo. Procurou-se caracterizar a equivalência entre os tipos de amostras através de análises químicas e medições de microdureza Vickers. As análises químicas foram executadas em um espectômetro de emissão ótica marca Spectrolab, modelo LAVMB08B. As medidas de microdureza foram realizadas em um microdurômetro marca Shimadzu, modelo HMV-2T.

Previamente a caracterização mecânica, todo o material (originalmente fornecido no estado T6) foi submetido a tratamentos térmicos para obtenção dos estados recozido e $\mathrm{T} 4$. Os tratamentos térmicos foram realizados em um forno elétrico tipo poço com circulação forçada de ar marca Sanchis, número 1.279, equipado com um medidor/controlador de temperatura marca Novus, modelo N1100, ligado a um termopar do tipo $\mathrm{K}$, sendo a precisão do conjunto de $\pm 1^{\circ} \mathrm{C}$. $\mathrm{O}$ tratamento de recozimento foi realizado mantendo-se o material por uma hora a $420^{\circ} \mathrm{C}$, seguido de resfriamento no forno até a temperatura ambiente. A taxa de resfriamento média foi de cerca de $50^{\circ} \mathrm{C}$ por minuto na faixa de 420 a $200^{\circ} \mathrm{C}$. O tratamento de solubilização foi realizado mantendo-se o material também por uma hora a $530^{\circ} \mathrm{C}$, seguido de resfriamento em água. $O$ envelhecimento foi realizado a temperatura ambiente. $O$ progresso do envelhecimento natural foi acompanhado realizando-se diariamente medições de dureza na escala Brinell. O envelhecimento foi considerado completo após sete dias quando houve a estabilização do valor de dureza. Para medição de dureza foi utilizado um durômetro marca Mitutoyo, com esfera de 2,5 mm de diâmetro e carga de $62,5 \mathrm{kgf}(612,5 \mathrm{~N})$. Todo o material submetido ao tratamento T4 foi antes submetido ao tratamento de recozimento previamente descrito.

Para o levantamento das curvas de escoamento foram utilizados corpos de prova cilíndricos com $10 \mathrm{~mm}$ de diâmetro e $15 \mathrm{~mm}$ de altura. Os ensaios foram realizados em uma máquina universal de ensaios mecânicos marca Schenck, modelo PC400M, dotada de acionamento hidráulico. A máquina possui uma capacidade de $400 \mathrm{kN}$ de força, curso de $100 \mathrm{~mm}$ e velocidade máxima do cilindro de $500 \mathrm{~mm} / \mathrm{s}$. O sistema de controle permite a realização de ensaios com velocidade de deformação constante. A força é medida por uma célula de carga marca HBM, modelo PM400Rn a base de extensômetros de resistência elétrica (strain-gauges) com precisão de $\pm 0,1 \%$. $O$ deslocamento é medido por um transdutor do tipo indutivo, marca HBM, modelo W100 com precisão de $\pm 1 \%$. Os sinais de deslocamento e força são tratados em dois condicionadores de sinal marca HBM, modelos MV317 e MV318, respectivamente. A precisão total do sistema é estimada em cerca de $\pm 0,2 \%$ para a força e $\pm 1,0 \%$ para o deslocamento. Este mesmo equipamento foi utilizado na realização dos ensaios para calibração do atrito, do dano acumulado, da instabilidade plástica e do processo de expansão.

O ensaio utilizado para calibração do atrito foi o teste do anel plano proposto por Male e Cockroft [7]. Foram utilizadas amostra com $8 \mathrm{~mm}$ de diâmetro externo e 4 $\mathrm{mm}$ de diâmetro interno e 2,7 mm de espessura. As amostras foram lubrificadas com um produto comercial a base de grafite em água (Aerodag $\mathrm{G}$ ) e as ferramentas de compressão com MoS 2 . Os ensaios foram realizados com a velocidade de $1 \mathrm{~mm} / \mathrm{s}$ e

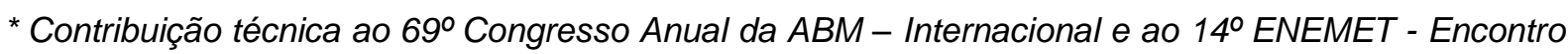
Nacional de Estudantes de Engenharia Metalúrgica, de Materiais e de Minas, 21 a 25 de julho de 2014, São Paulo, SP, Brasil. 


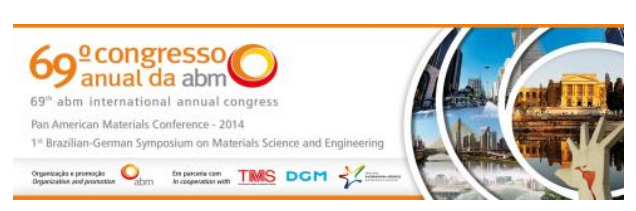

deformações relativas em altura de 18, 37 e 56\%. Um paquímetro foi utilizado para medição das espessuras dos anéis deformados. Os diâmetros internos, por outro lado, foram medidos em um projetor de perfis marca Mitutoyo, modelo PJ $300 \mathrm{H}$, com aumento de 10 vezes, utilizando dispositivos eletrônicos de medição do próprio projetor. Para determinação dos valores de atrito foram calculadas curvas de calibração através do método dos elementos finitos, com a utilização do programa IFORM 2D, versão 27. Foram calculadas curvas para o modelo do fator de atrito constante de Prandtl [8] e para o modelo geral de atrito de Wanheim-Bay [9,10]. Foram também realizados teste sem nenhum tipo de lubrificação.

A caracterização do material quanto a deformabilidade (ou determinação do dano crítico), foi realizada através de uma técnica de calibração inversa, para o critério de fratura dúctil de Cockcroft-Latham [11], com simulações realizadas com o mesmo programa utilizado anteriormente para calibração do teste do anel. Nos ensaios foram utilizados corpos de prova cilíndricos com $10 \mathrm{~mm}$ de diâmetro e $25 \mathrm{~mm}$ de altura. Os corpos de prova foram comprimidos em incrementos de 0,5 em 0,5 mm entre placas planas, sendo que foram utilizados calços para garantir a precisão dos incrementos de deformação. Antes do início dos ensaios as placas de compressão e os corpos de prova foram limpos com álcool isopropílico. Após a aplicação de cada incremento de deformação os corpos de prova foram examinados através de ensaio de líquidos penetrantes, sob aumento de 50 vezes, visando determinar a existência de trincas na superfície lateral dos mesmos. Os ensaios foram realizados a temperatura ambiente, com velocidade de compressão de cerca de $1,0 \mathrm{~mm} / \mathrm{s}$.

Para caracterização do comportamento dos tubos quanto à instabilidade (flambagem) foram utilizadas amostras da liga ABNT 6061, nos três estados de tratamentos térmicos. Foram utilizadas amostras com $90 \mathrm{~mm}$ de comprimento, sendo que os tubos tem diâmetro externo de $50,8 \mathrm{~mm}$ e espessura da parede de 2 $\mathrm{mm}$. Os tubos foram comprimidos entre placas planas sendo a força e o deslocamento da placa superior registrados durante o processo. A tensão crítica para instabilidade foi determinada dividindo-se a força registrada no início da ocorrência da flambagem (pico da curva) pela área da seção transversal do tubo. Os valores obtidos experimentalmente foram comparados a valores obtidos em simulações numéricas realizadas com programa IFORM 2D e com a tensão teórica de instabilidade calculada utilizando-se a equação (Equação 1) desenvolvida por Timoshenko [12] com base no conceito de módulo de tangência de Engesser-Shanley [12]:

$$
\sigma_{c r}=\frac{1}{\sqrt{3 \cdot\left(1-v^{2}\right)}} E_{t} \frac{t_{0}}{r}
$$

em que $v$ é coeficiente de Poisson, $E_{t}$ é o modulo de tangência, e to e rsão a espessura e o raio interno do tubo, respectivamente.

Os ensaios de expansão de extremidades dos tubos foram realizados utilizando-se os punções (matriz superior) vistos na Figura 2, sendo uma placa plana utilizada como matriz inferior. Para o estudo computacional foi montado um plano de simulações considerando (Figura 1): a relação $r_{p} / r_{0}$, ângulo $\alpha$ e o regime de lubrificação. A Tabela 1 mostra as condições de realização dos ensaios de expansão. Na tabela também são resumidos os resultados obtidos: instabilidade (I), expansão (E) ou ruptura (R).

\footnotetext{
* Contribuição técnica ao $69^{\circ}$ Congresso Anual da ABM - Internacional e ao 14ํㅡㄹ ENEMET - Encontro Nacional de Estudantes de Engenharia Metalúrgica, de Materiais e de Minas, 21 a 25 de julho de 2014, São Paulo, SP, Brasil.
} 

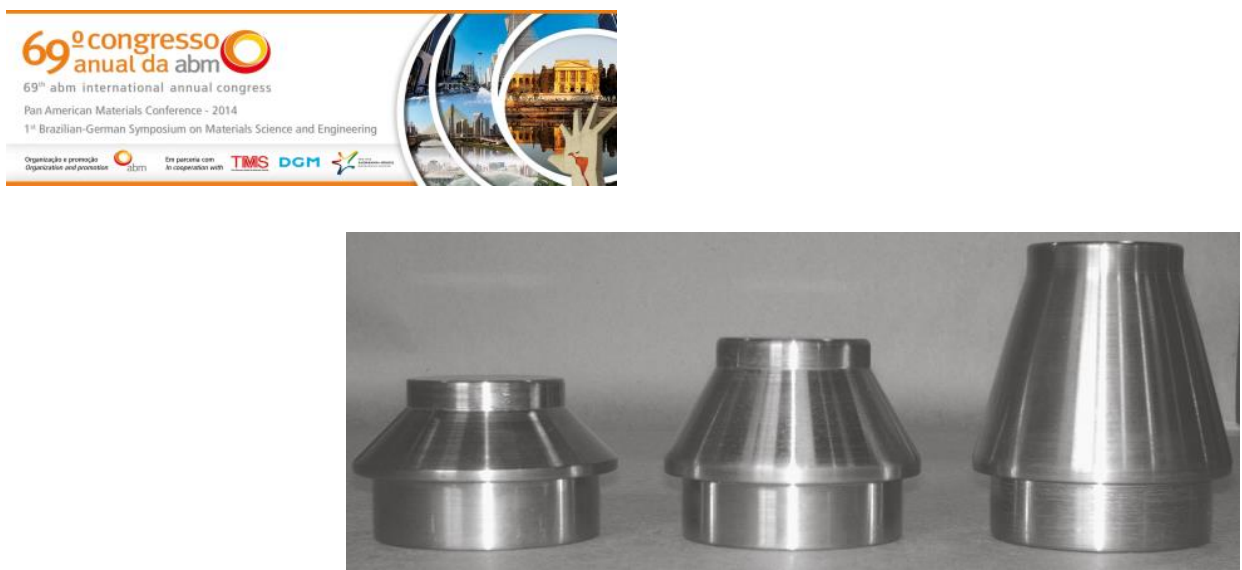

Figura 2. Punções utilizados nos ensaios de expansão.

Tabela 1. Casos estudados no processo de expansão

\begin{tabular}{|c|c|c|c|c|c|}
\hline Casos & Lubrificação & $\alpha(\stackrel{o}{)})$ & $r_{p}(\mathrm{~mm})$ & $r_{p} / r_{0}$ & Resultados \\
\hline $1(0) / 1(\mathrm{~T} 4) / 1(\mathrm{~T} 6)$ & sem lubrificação & 15 & 28,20 & 1,20 & $\mathrm{E} / \mathrm{E} / \mathrm{E}$ \\
\hline 2(0) / 2(T4) / 2(T6) & Aerodag $\mathrm{G}+\mathrm{MoS}_{2}$ & 15 & 28,20 & 1,20 & $E / E / E$ \\
\hline $3(0) / 3(\mathrm{~T} 4) / 3(\mathrm{~T} 6)$ & sem lubrificação & 15 & 31,59 & 1,35 & $\mathrm{I} / \mathrm{I} / \mathrm{E}$ \\
\hline $4(0) / 4(\mathrm{~T} 4) / 4(\mathrm{~T} 6)$ & Aerodag $\mathrm{G}+\mathrm{MoS}_{2}$ & 15 & 31,59 & 1,35 & $E / E / E$ \\
\hline $5(0) / 5(T 4) / 5(T 6)$ & sem lubrificação & 15 & 35,10 & 1,50 & $\mathrm{R} / \mathrm{R} / \mathrm{R}$ \\
\hline 6(0) / 6(T4) / 6(T6) & Aerodag $\mathrm{G}+\mathrm{MoS}_{2}$ & 15 & 35,10 & 1,50 & $\mathrm{R} / \mathrm{R} / \mathrm{R}$ \\
\hline $7(0) / 7(\mathrm{~T} 4) / 7(\mathrm{~T} 6)$ & sem lubrificação & 30 & 28,20 & 1,20 & $E / E / E$ \\
\hline $8(0) / 8(T 4) / 8(T 6)$ & Aerodag G $+\mathrm{MoS}_{2}$ & 30 & 28,20 & 1,20 & $E / E / E$ \\
\hline 9(0) / 9(T4) / 9(T6) & sem lubrificação & 30 & 31,59 & 1,35 & $\mathrm{I} / \mathrm{I} / \mathrm{E}$ \\
\hline $10(0) / 10(\mathrm{~T} 4) / 10(\mathrm{~T} 6)$ & Aerodag $\mathrm{G}+\mathrm{MoS}_{2}$ & 30 & 31,59 & 1,35 & $E / E / E$ \\
\hline $11(0) / 11(\mathrm{~T} 4) / 11(\mathrm{~T} 6)$ & sem lubrificação & 30 & 35,10 & 1,50 & $\mathrm{I} / \mathrm{I} / \mathrm{R}$ \\
\hline $12(0) / 12(\mathrm{~T} 4) / 12(\mathrm{~T} 6)$ & Aerodag $\mathrm{G}+\mathrm{MoS}_{2}$ & 30 & 35,10 & 1,50 & $\mathrm{I} / \mathrm{I} / \mathrm{R}$ \\
\hline 13(0) / 13(T4) / 13(T6) & sem lubrificação & 45 & 28,20 & 1,20 & $\mathrm{I} / \mathrm{I} / \mathrm{E}$ \\
\hline $14(0) / 14(\mathrm{~T} 4) / 14(\mathrm{~T} 6)$ & Aerodag G $+\mathrm{MoS}_{2}$ & 45 & 28,20 & 1,20 & $E / E / E$ \\
\hline $15(0) / 15(\mathrm{~T} 4) / 15(\mathrm{~T} 6)$ & sem lubrificação & 45 & 31,59 & 1,35 & $1 / 1 / 1$ \\
\hline 16(0) / 16(T4) / 16(T6) & Aerodag $\mathrm{G}+\mathrm{MoS}_{2}$ & 45 & 31,59 & 1,35 & $1 / I / I$ \\
\hline 17(0) / 17(T4) / 17(T6) & sem lubrificação & 45 & 35,10 & 1,50 & $\mathrm{I} / \mathrm{I} / \mathrm{I}$ \\
\hline 18(0) / 18(T4) / 18(T6) & Aerodag $\mathrm{G}+\mathrm{MoS}_{2}$ & 45 & 35,10 & 1,50 & $\mathrm{I} / \mathrm{I} / \mathrm{I}$ \\
\hline
\end{tabular}

\section{RESULTADOS E DISCUSSÃO}

\subsection{Caracterização do Material}

A Tabela 2 mostra a análise química do material nos dois formatos utilizados, ou seja, tubos e barra extrudada. A Figura 3 mostra os perfis de microdureza Vickers obtidos para os dois casos em seções transversais e longitudinais. Analisando a tabela e a figura verifica-se que não há diferenças significativas entre os materiais.

Tabela 2. Análise química dos materiais utilizados

\begin{tabular}{|lcccccccc|}
\hline Elemento & $\mathrm{Al}$ & $\mathrm{Mg}$ & $\mathrm{Si}$ & $\mathrm{Cu}$ & $\mathrm{Fe}$ & $\mathrm{Mn}$ & $\mathrm{Zn}$ & Outros \\
\hline $\begin{array}{l}\text { \% em massa } \\
\text { (barra) }\end{array}$ & 97,84 & 1,0 & 0,51 & 0,12 & 0,22 & 0,08 & 0,10 & 0,13 \\
\hline$\%$ em massa (tubo) & 98,04 & 0,9 & 0,47 & 0,10 & 0,23 & 0,03 & 0,12 & 0,11 \\
\hline
\end{tabular}

A Tabela 3 mostra as curvas de escoamento, apresentadas na forma de uma equação de Ludwik-Hollomon [12], onde $k_{f}$ é a tensão de escoamento e $\mathbf{f}$ a deformação verdadeira e a velocidade de deformação é igual a 1,0/s. Os valores do dano crítico e da tensão crítica de instabilidade obtidos conforme descrito anteriormente são apresentados na mesma tabela. A Tabela 4 apresenta os valores de atrito para os casos calibrados.

\footnotetext{
* Contribuição técnica ao 69ำ Congresso Anual da ABM - Internacional e ao 14ํㅡㄹ ENEMET - Encontro Nacional de Estudantes de Engenharia Metalúrgica, de Materiais e de Minas, 21 a 25 de julho de 2014, São Paulo, SP, Brasil.
} 

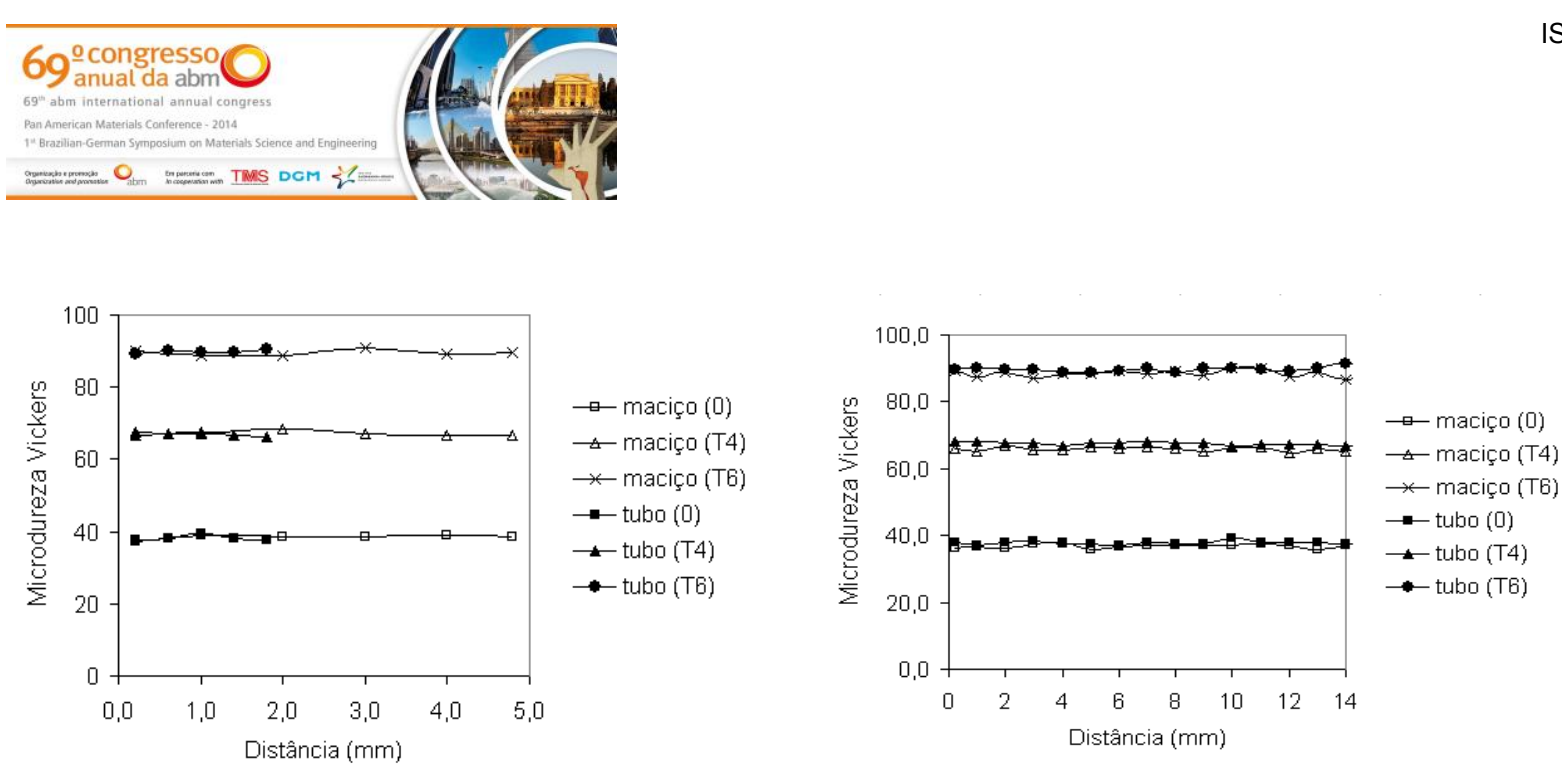

Figura 3. Perfis de microdureza Vickers nas seções transversais (à esquerda) e longitudinais (à direita) dos tubos e barras (maciço) em diferentes condições de tratamentos térmicos.

Tabela 3. Curvas de escoamento e dano crítico em função do tratamento térmico

\begin{tabular}{|c|c|c|c|}
\hline $\begin{array}{l}\text { Tratamento } \\
\text { térmico }\end{array}$ & $\begin{array}{l}\text { Curva de escoamento } \\
(\mathrm{MPa})\end{array}$ & $\begin{array}{l}\text { Dano } \\
\text { crítico }\end{array}$ & $\begin{array}{l}\text { Tensão crítica de } \\
\text { instabilidade, (MPa) }\end{array}$ \\
\hline Condição "0" & $k_{f}=125,95 \cdot \varphi^{0,704} ; k_{f \mid \varphi=0}=51,0$ & 0,5574 & 55,56 \\
\hline Condição T4 & $k_{f}=228,30 \cdot \varphi^{0,143} ; k_{f \mid \varphi=0}=106,8$ & 0,4088 & 111,90 \\
\hline Condição T6 & $k_{f}=209,19 \cdot \varphi^{0,032} ; k_{f \mid \varphi=0}=176,0$ & 0,3128 & 170,40 \\
\hline
\end{tabular}

Tabela 4. Valores de atrito calibrados

\begin{tabular}{|c|c|c|c|c|}
\hline $\begin{array}{c}\text { Tratamento } \\
\text { térmico }\end{array}$ & \multicolumn{2}{|c|}{ Sem lubrificação } & \multicolumn{2}{c|}{ Aerodag $\mathrm{G}+\mathrm{MoS}_{2}$} \\
\cline { 2 - 5 } Condição "0" & 0,3 & 0,6 & 0,1 & 0,4 \\
\hline Condição T4 & 0,3 & 0,5 & 0,05 & 0,2 \\
\hline Condição T6 & 0,3 & 0,6 & 0,05 & 0,2 \\
\hline
\end{tabular}

Os resultados apresentados na Tabela 2 e na Figura 3 mostram que os materiais (barra e tubo) são bastante semelhantes. Assim, a utilização das propriedades obtidas em ensaios realizados com amostras retiradas das barras em simulações numéricas do processo de expansão dos tubos é, provavelmente, possível sem a introdução de erros significativos nos resultados da simulação. Além disso, os valores apresentados são compatíveis com valores encontrados na literatura [13]. Quanto a Tabela 3, os resultados apresentados também são compatíveis com valores existentes na literatura [13-15]. Além disso, os valores são compatíveis com os tratamentos térmicos a que o material foi submetido, ou seja, resistência do material recozido é menor que a do material no estado T4, que é menor que a do material no estado T6, pelo menos para baixas deformações antes que ocorra um encruamento significativo. Quanto aos resultados de atrito apresentados na Tabela 4, é difícil encontrar valores equivalentes na literatura para comparação já que os valores são calibrados para o sistema material/matriz/condições do ensaio. Entretanto os valores, tanto de $m$ quanto de $f$, são significativamente menores para os ensaios realizados com lubrificação.

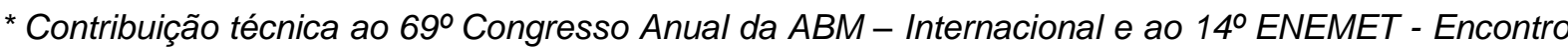
Nacional de Estudantes de Engenharia Metalúrgica, de Materiais e de Minas, 21 a 25 de julho de 2014, São Paulo, SP, Brasil.
} 


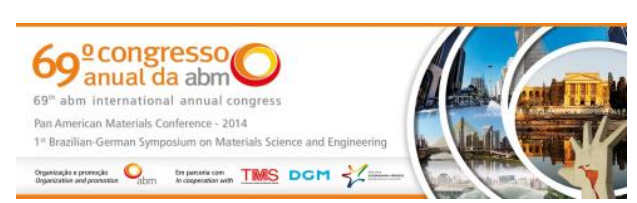

\subsection{Testes de Expansão}

\subsubsection{Modos de deformação}

O trabalho experimental sobre expansão de tubos confirmou a existência de três diferentes modos de deformação. Para relações de $r_{p} / r_{0}$ grandes e pequenos comprimentos de expansão (altos valores do ângulo a) a deformabilidade está limitada pela ocorrência de instabilidade plástica. Por outro lado para grandes comprimentos de expansão ( $\alpha$ pequenos) o limite de deformabilidade é a ocorrência de fratura dúctil nas regiões altamente tracionadas na direção circunferencial. A Figura 4 mostra três dos espécimes ensaiados, cada um apresentando um modo de deformação.

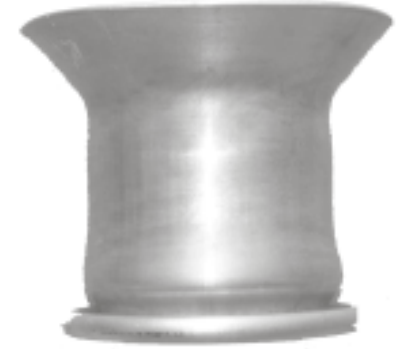

(a)

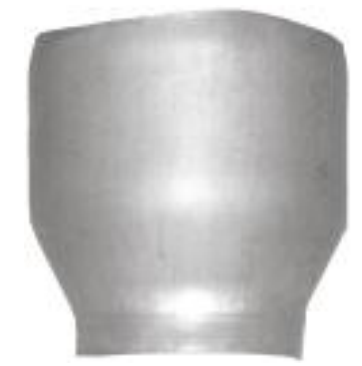

(b)

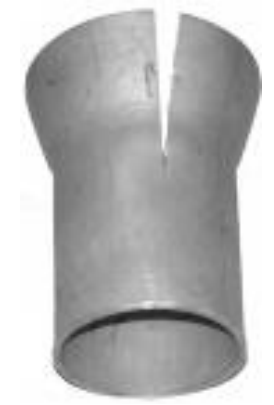

(c)

Figura 4. Modos de deformação observados no estudo do processo de expansão: (a) instabilidade (b) expansão e (c) ruptura

\subsubsection{Lubrificação}

A Figura 5 mostra como um processo bem sucedido de expansão pode falhar simplesmente por uma variação no regime de lubrificação. Um exame detalhado das curvas força-deslocamento experimental e prevista pela simulação numérica mostradas nessa figura, para o processo de expansão, permite identificar três diferentes etapas de conformação: (a) compressão axial; (b) expansão (transiente); e, (c) expansão em regime permanente ou instabilidade localizada. Na primeira etapa a força aumenta, quase linearmente com o avanço do punção na extremidade do tubo, até um valor de cerca de $13 \mathrm{kN}$ para ambos os casos (sem e com lubrificação). Após esse ponto o tubo começa a dobrar axialmente de modo a ajustar-se a superfície cônica do punção. Subsequentemente (segundo estágio) a borda do tubo começa a escoar ao logo da superfície cônica, ocorrendo um progressivo aumento das tensões de tração circunferenciais, resultando em um aumento da força. A terceira e última etapa é diferente para cada um dos casos. No caso com lubrificação a expansão do tubo passa a ocorrer em regime permanente, após o "endireitamento" (ver Figura 1), com uma força aproximadamente constante, igual a $32 \mathrm{kN}$. No caso sem lubrificação desenvolve-se um modo de deformação caracterizado por instabilidade plástica antes que a borda do tubo atinja o fim da zona cônica do punção. Isto ocorre porque nesse caso o nível de carregamento é muito maior, consequência do aumento do atrito que faz com que a força de conformação acabe por atingir a carga crítica de instabilidade, que ocorre em cerca de $39 \mathrm{kN}$.

\footnotetext{
* Contribuição técnica ao $69^{\circ}$ Congresso Anual da ABM - Internacional e ao 14ํㅡㄹ ENEMET - Encontro Nacional de Estudantes de Engenharia Metalúrgica, de Materiais e de Minas, 21 a 25 de julho de 2014, São Paulo, SP, Brasil.
} 

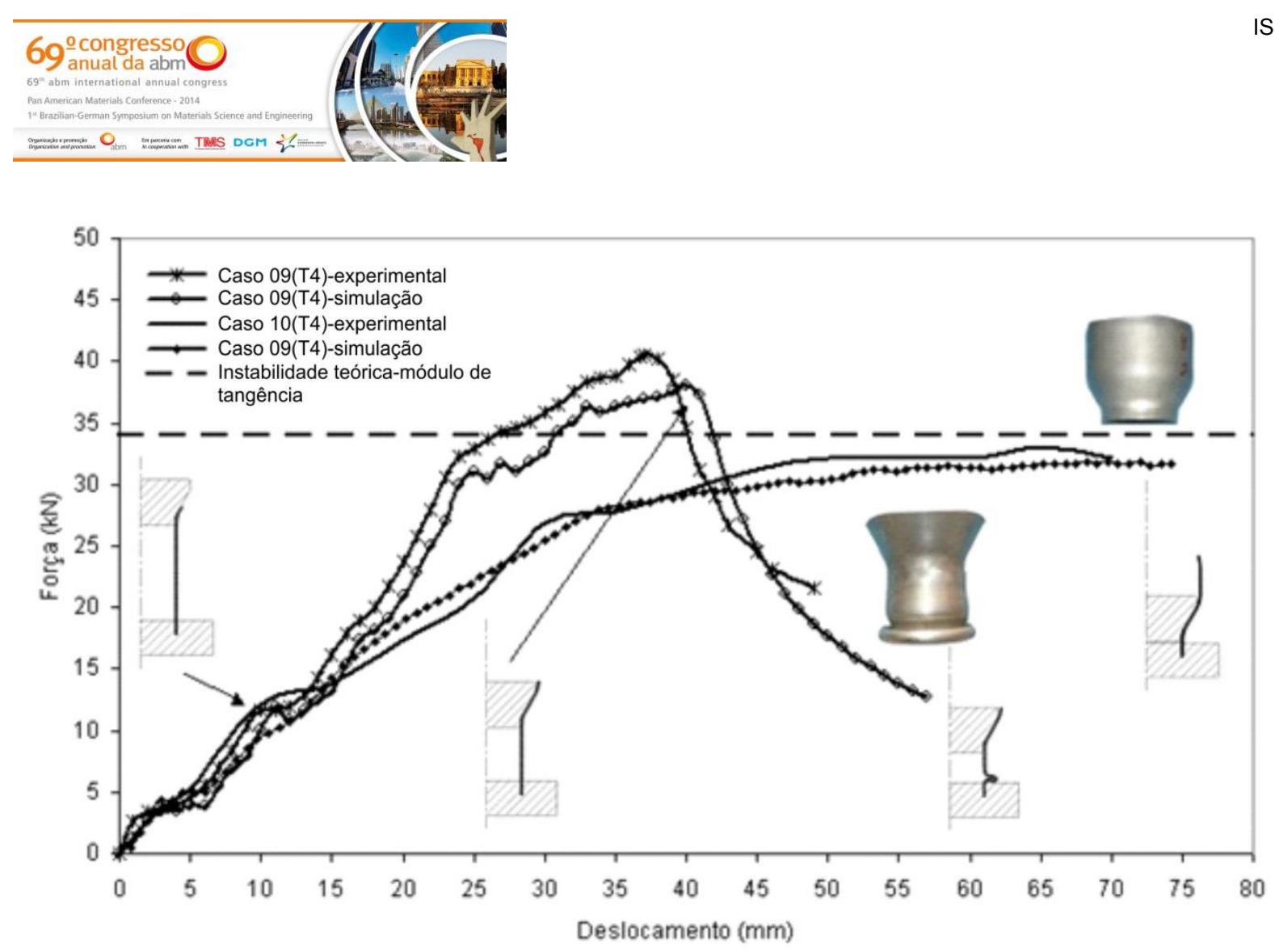

Figura 5. Curvas força-deslocamento simuladas e experimentais e aspecto geral das amostras deformadas em função do regime de lubrificação. Casos 09(T4) e 10(T4) da Tabela 1.

A comparação entre as curvas força-deslocamento obtidas experimentalmente e através de simulação para diferentes processos de expansão mostrou que os valores de fator de atrito para os quais as mesmas melhor coincidem são $m=0,08$ para os casos sem lubrificação e $m=0,02$ para os com lubrificação. $O$ pequeno valor do fator de atrito para a expansão de tubos confirma a inadequação do uso da lei do atrito constante para o modelamento numérico de processos com baixas pressões de contato devido ao fato das tensões de atrito serem superestimadas. Visto que $o$ fator de atrito, $m$, determinado pelo teste do anel, para o caso sem lubrificação, foi encontrado ser igual a 0,3 independentemente da condição do material, fica também evidente a inadequação desse método para caracterização das condições tribológicas na interface de contato entre o tubo e o punção de expansão.

\subsubsection{Dano e instabilidade}

A distribuição do dano para o caso 6 (T6) da Tabela $1\left(r_{p} / r_{0}=1,50, \alpha=15^{\circ}\right.$, lubrificação com Aerodag $\mathrm{G}+\mathrm{MoS}_{2}$ ) é mostrada na Figura 6. Esse resultado foi obtido através de uma análise não acoplada baseada no critério normalizado de fratura dúctil de Cockcroft-Latham [12]. Em uma análise não acoplada a influência do dano nas equações constitutivas não é considerada, sendo o material assumido como contínuo e isotrópico. As previsões do dano pela simulação não apenas corroboram as observações experimentais quanto à localização da trinca (borda do tubo) como também o nível de deformação onde a mesma tem início. Resultados similares foram encontrados para os casos 6 (0) e 6 (T4) de acordo com os valores de dano calibrados para cada condição do material.

\footnotetext{
* Contribuição técnica ao $69^{\circ}$ Congresso Anual da ABM - Internacional e ao 14ํㅡㄹ ENEMET - Encontro Nacional de Estudantes de Engenharia Metalúrgica, de Materiais e de Minas, 21 a 25 de julho de 2014, São Paulo, SP, Brasil.
} 


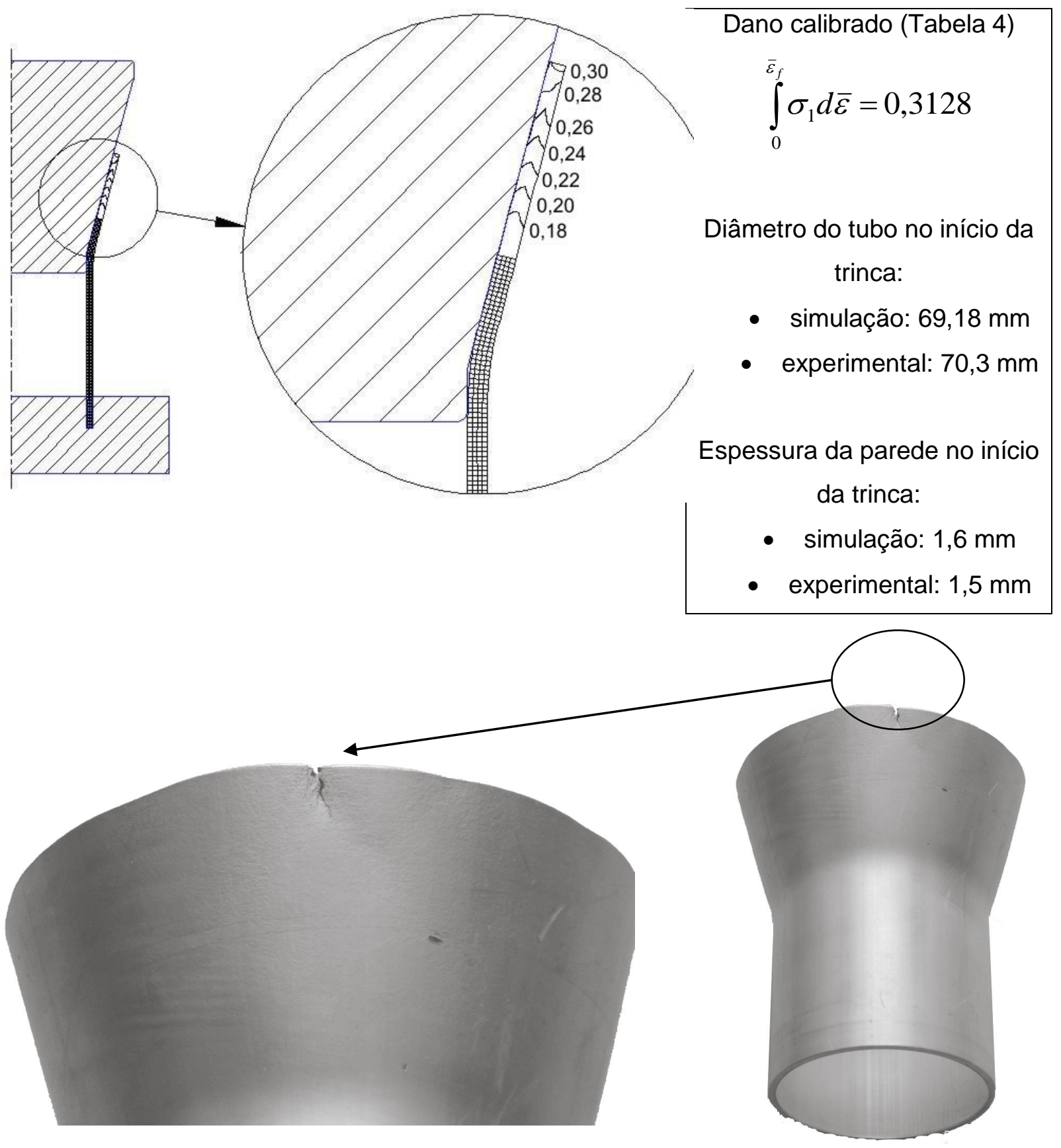

Figura 6. Distribuição do dano para o caso 24 (T6) da tabela $5.5\left(r_{p} / r_{0}=1,50, \quad \alpha=15^{\circ}\right.$, lubrificação com Aerodag $G+M_{2}$ ) simulada segundo o critério de fratura dúctil de Cockcroft-Latham e resultado experimental.

A Figura 7 mostra curvas força-deformação obtidas através da simulação e experimentalmente para cada caso. No que diz respeito ao resultado da simulação numérica é importante mencionar que o aumento contínuo da força além do nível de deformação onde a trinca se inicia é consequência do cálculo do dano através de um modelo numérico não acoplado. A ocorrência de ruptura para o material no estado T6 também foi prevista em simulação 3D realizada com o programa QFORM. Embora o programa não tenha modelos de dano implementados é capaz de sugerir a ocorrência de trincas com base na deformação equivalente calculada. Esse resultado é visto na figura 8 . Os valores calculados pelo programa para o diâmetro

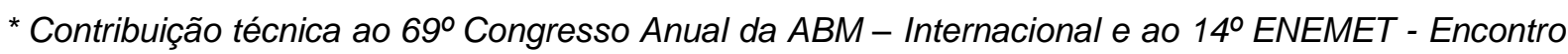
Nacional de Estudantes de Engenharia Metalúrgica, de Materiais e de Minas, 21 a 25 de julho de 2014, São Paulo, SP, Brasil.
} 

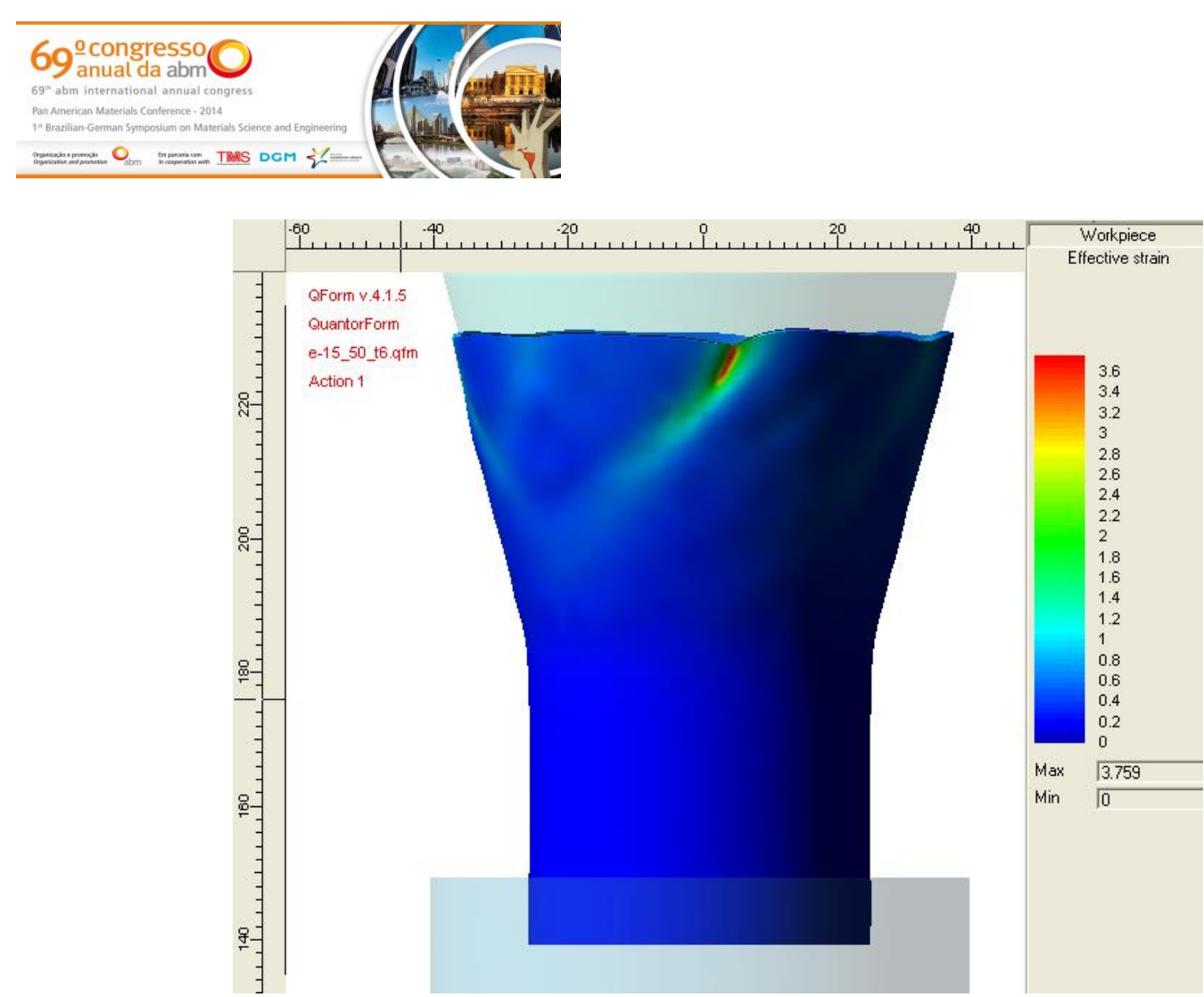

Figura 8. Previsão de trinca feita através de simulação 3D no programa QFORM para o caso 6(T6) da tabela $1\left(r_{p} / r_{0}=1,50\right.$; sem lubrificação).

$\mathrm{Na}$ questão da caracterização tribológica o pequeno valor de atrito encontrado nos testes com tubos confirma a inadequação do uso da lei do atrito constante (Prandtl) para o modelamento numérico de processos com baixas pressões de contato devido a uma superestimativa das tensões de atrito. Também ficou evidente a inadequação da utilização do teste do anel para determinação do valor do atrito para esses casos. A utilização da metodologia empregada na determinação do dano crítico e da carga crítica que leva a instabilidade dos tubos também se mostrou adequada. Os dois programas utilizados para simulação foram capazes de prever a ocorrência de trincas para valores de dano (Cockcroft-Latham) muito próximos dos verificados experimentalmente. $\mathrm{O}$ mesmo se aplica à questão da carga crítica de instabilidade $\mathrm{e}$ à forma da instabilidade.

O processo de expansão de tubos usando uma matriz mostrou ser factível apenas dentro de uma estreita faixa de parâmetros processuais. Ficou demonstrado que a simulação numérica por elementos finitos, aliada a informações sobre valores de dano que levam à ocorrência de fratura, pode ser um método de grande ajuda no desenvolvimento de processos de expansão.

\section{Agradecimentos}

Os autores agradecem ao Prof. Dr. Paulo António Firme Martins da Universidade Técnica de Lisboa pela cessão dos direitos de uso do programa IFORM 2D. Ao Dr. Nikolay Biba da empresa Quantor Form Ltd. Pela cessão dos direitos de uso do programa QFORM 3D. A CAPES pelo apoio financeiro no projeto de Cooperação Internacional Brasil/Portugal CAPES/GRICES no 133/05.

\footnotetext{
* Contribuição técnica ao 69ำ Congresso Anual da ABM - Internacional e ao 14ํㅡㄹ ENEMET - Encontro Nacional de Estudantes de Engenharia Metalúrgica, de Materiais e de Minas, 21 a 25 de julho de 2014, São Paulo, SP, Brasil.
} 


\section{REFERÊNCIAS}

1 Rosa PAR, Rodrigues JMC, Martins PAF. External invertion of thin-walled tubes using a die: experimental and theoretical investigation. Journal of Machine Tools and Manufacture. 2003;43(8):787-796.

2 Rosa PAR, Baptista MO, Rodrigues J MC, Martins PAF. An investigation on the external inversion of thin-walled tubes using a die. International Journal of Plasticity. 2004;20(10):1931-46.

3 Rosa PAR, Rodrigues JMC, Martins PAF. Internal inversion of thin-walled tubes using a die: experimental and theoretical investigation. Internacional Journal of Machine Tools and Manufacture. 2004;44(7-8):775-784.

4 Avitzur B. Tube sinkig and expanding. Journal of Engineering for Industry. 1965;87(1):71-79.

5 Sadok L, Kusiak J, Packo M, Ruminski M. State of strain in the tube sinking process. Journal of Materials Processing Technology. 1996;60(1-4):161-166.

6 Almeida BPP, Alves ML, Rosa PAR, Brito AMG, Martins PAF. Expansion and reduction of thin-walled tubes using a die: experimental an theoretical investigation. International Journal of Machine Tools and Manufacture. 2006;46(12-13):1643-52.

7 Male AT, Cockcroft MG. Method for the determination of the coeficient of friction of metal under conditions of bulk plastic deformation. Journal of the Institute of Metals. 1964;93(2):38-46.

8 Rodrigue JMC, MARTINS, PAF. Tecnologia mecânica: tecnologia da deformação plástica, vol. 1 - fundamentos teóricos. Lisboa: Escolar Editora; 2005.

9 Bay N, Wanheim T. Real area of contact and friction stress at high pressure sliding contact. Wear. 1976;38(2):201-206.

10 Petersen SB, Martins PAF, Bay N. Friction in bulk metal forming: a general friction model vs. the law of constant friction. Journal of Materials Processing Technology. 1997;66(1-3):186-194.

11 Cockcroft MG, Latham DJ. Ductility and the workability of metals. Journal of the Institute of Metals. 1968;96(1):33-39.

12 Brito AMG. Análise teórico-experimental dos processos de expansão, redução e inversão de extremidades de tubos de parede fina em matriz [doutor]. Porto Alegre: Universidade Federal do Rio Grande do Sul; 2006.

13 Rosa PAR. Inversão externa de tubos metálicos de parede fina. Lisboa: Universidade Técnica de Lisboa; 2003.

14 Altan T, Oh SI, Gegel H. Conformação de metais: fundamentos e aplicações. São Paulo: EESC, USP; 1999.

15 Hensel A, Spittel T. Kraft- und Arbeitsbedarf bildsamer Formgebungs-verfahren. Leipzig: VEB Deutscher Verlag; 1978.

\footnotetext{
* Contribuição técnica ao $69^{\circ}$ Congresso Anual da ABM - Internacional e ao 14ํㅡㄹ ENEMET - Encontro Nacional de Estudantes de Engenharia Metalúrgica, de Materiais e de Minas, 21 a 25 de julho de 2014, São Paulo, SP, Brasil.
} 